\title{
THE FATOU THEOREM FOR FUNCTIONS HARMONIC IN A HALF-SPACE
}

\author{
By F. W. GEHRING
}

[Received 6 May 1957.-Read 16 May 1957]

\section{Introduction}

WE say that $u(x, y, z)$ is in $H^{+}$if $u(x, y, z)$ is harmonic and non-negative for $z>0$. We say that $u(x, y, z)$ is in $H$ if $u(x, y, z)$ can be expressed as the difference of two functions, each of which is in $H^{+}$. For functions in $H$ we have the following known result. (See (8) 9.)

Lemma 1. The function $u(x, y, z)$ is in $H$ if and only if it has the representation

$$
u(x, y, z)=k z+\frac{z}{2 \pi} \int_{-\infty}^{\infty} \int_{[}^{\left[(x-\xi)^{2}+(y-\eta)^{2}+z^{2}\right]^{\frac{\pi}{2}}}
$$

where $k$ is a constant and where $\mu$ is a measure defined for all Borel sets in the $z$-plane such that

$$
\int_{-\infty}^{\infty} \int_{\infty} \frac{|d \mu(\xi, \eta)|}{\left[\xi^{2}+\eta^{2}+1\right]^{\frac{\beta}{2}}}<\infty .
$$

Furthermore, $u(x, y, z)$ is in $H^{+}$if and only if $k$ and $\mu$ are non-negative.

If $\mu$ satisfies (1.2) then, for each $\epsilon>0$, it is not difficult to show that

$$
\frac{z}{2 \pi} \int_{\xi^{2}+\eta^{2} \geqslant \epsilon^{2}} \frac{d \mu(\xi, \eta)}{\left[(x-\xi)^{2}+(y-\eta)^{2}+z^{2}\right]^{\frac{a}{2}}}=O(z)
$$

as $(x, y, z) \rightarrow(0,0,0)$ along any path in $z>0$.

Definition. Suppose that $\mu$ is a measure defined for all Borel sets in the plane. We say that $D \mu(0,0)=A$ if

$$
\frac{1}{\pi t^{2}} \int_{0}^{2 \pi} \int_{0}^{t} d \mu(r, \theta) \rightarrow A
$$

as $t \rightarrow 0+$. We say that $D^{*} \mu(0,0)=A$ if

$$
\frac{1}{\pi t^{2}} \int_{\alpha}^{\beta} \int_{0}^{t} d \mu(r, \theta) \rightarrow \frac{\beta-\alpha}{2 \pi} A
$$

Proc. London Math. Soc. (3) 8 (1958) 
as $t \rightarrow 0+$ for all $\alpha<\beta$ and if there exists a constant $B$ such that

$$
\left|\frac{1}{\pi t^{2}} \int_{\alpha}^{\beta} \int_{0}^{t} d \mu(r, \theta)\right| \leqslant B<\infty
$$

for all $\alpha<\beta \leqslant \alpha+2 \pi$ and all small $t$; when $\mu$ is non-negative, (1.5) implies (1.6). Obviously $D^{*} \mu(0,0)=A$ implies that $D \mu(0,0)=A$.

In this paper we are concerned with the behaviour of the function $u(x, y, z)$ near $(0,0,0)$ and the behaviour of the measure $\mu$ near $(0,0)$. In particular we prove the following theorems which are three-dimensional analogues of results due to Fatou ((15) 52-53) and Loomis (9).

Theorem 1. Suppose that $u(x, y, z)$ in $H$ is given by (1.1) and that

$$
D_{\mu}(0,0)=A \text {. }
$$

Then

$$
u(0,0, z) \rightarrow A
$$

as $z \rightarrow 0+$.

Theorem 2. Suppose that $u(x, y, z)$ in $H^{+}$is given by (1.1) and that

$$
u(0,0, z) \rightarrow A
$$

as $z \rightarrow 0+$. Then $D_{\mu}(0,0)=A$.

Theorem 3. Suppose that $u(x, y, z)$ in $H$ is given by (1.1) and that

$$
D^{*} \mu(0,0)=A \text {. }
$$

Then

$$
u(x, y, z) \rightarrow A
$$

as $(x, y, z) \rightarrow(0,0,0)$ along each ray in $z>0$.

Theorem 4. Suppose that $u(x, y, z)$ in $H^{+}$is given by (1.1) and that

$$
u(x, y, z) \rightarrow A
$$

as $(x, y, z) \rightarrow(0,0,0)$ along each ray in $z>0$. Then $D^{*} \mu(0,0)=A$.

Theorems 1 and 3 are Abelian theorems and Theorems 2 and 4 are the corresponding 'partial converses' or Tauberian theorems. The Tauberian condition is that $u(x, y, z) \geqslant 0$.

2. Proofs for Theorem 1 and Theorem 2

Obviously we can assume that $k=0$ in (1.1). Next set

$$
\mu(r, \theta)=\int_{0}^{\theta} \int_{0}^{r} d \mu(r, \theta)
$$

and make the change of variables

$$
\zeta=z^{2}, \quad \rho=r^{2}, \quad \gamma(\rho)=\mu(r, 2 \pi) .
$$


We see from (1.1) that

$$
u(0,0, z)=\frac{\zeta^{\frac{1}{z}}}{2 \pi} \int_{0}^{\infty} \frac{d \gamma(\rho)}{(\rho+\zeta)^{\frac{3}{2}}}
$$

for each $z>0$. Now the hypotheses of Theorem 1, (2.1) and (2.2) imply that

$$
\gamma(\rho) \sim \pi A \rho
$$

as $\rho \rightarrow 0+$ and we can apply a well-known Abelian theorem for the Stieltjes transform ((14) 185) to conclude that

$$
\int_{0}^{\infty} \frac{d \gamma(\rho)}{(\rho+\zeta)^{\frac{3}{2}}} \sim \frac{2 \pi A}{\zeta^{\frac{1}{2}}}
$$

as $\zeta \rightarrow 0+$. This, together with (2.3), completes the proof for Theorem 1 .

Consider Theorem 2. The fact that $u(x, y, z)$ is in $H^{+}$means that $\mu$ is non-negative and hence that $\gamma(\rho)$ is non-decreasing in $\rho$. Furthermore, the hypotheses of Theorem 2 and (2.3) imply that (2.5) holds and we can apply the Stieltjes integral form of a Tauberian theorem due to Hardy and Littlewood ((6) 25 or (9) 242) to conclude that (2.4) holds. This, together with (2.2) and (2.1), completes the proof for Theorem 2.

\section{Proof for Theorem 3}

First observe that we can assume $A=0$. For if we define

$$
\mu_{1}(E)=\mu(E)-A \iint_{E} d \xi d \eta
$$

and let $u_{1}(x, y, z)$ be the corresponding harmonic function given by (1.1), then $D^{*} \mu_{1}(0,0)=0$ and

$$
u_{1}(x, y, z)=u(x, y, z)-A
$$

for all $(x, y, z)$ in $z>0$. Furthermore, we can assume that $k=0$ and, by virtue of (1.3), that $d \mu=0$ outside of the circle $\xi^{2}+\eta^{2} \leqslant \epsilon^{2}$.

If in (1.1) we introduce spherical coordinates $(t, \psi, \phi)$ for $(x, y, z)$ and polar coordinates $(r, \theta)$ for $(\xi, \eta)$, then we obtain

$$
u(x, y, z)=u(t, \psi, \phi)=\frac{1}{t^{2}} \int_{0}^{2 \pi} \int_{0}^{\infty} \lambda(r / t, \psi, \theta-\phi) d \mu(r, \theta)
$$

where

$$
\lambda(r, \psi, \theta)=\frac{1}{2 \pi} \frac{\cos \psi}{\left[r^{2}-2 r \sin \psi \cos \theta+1\right]^{\sharp}} .
$$

For each fixed $\phi$ and $\psi$ we want to show that

$$
u(t, \psi, \phi) \rightarrow 0
$$


as $t \rightarrow 0+$. Obviously we may assume for convenience that $\phi=0$. Then if we replace $r$ by $r t$ the integral in (3.1) can be written as

$$
\frac{1}{t^{2}} \int_{0}^{2 \pi} \int_{0}^{\infty}\{\lambda(r, \psi, \theta)-\lambda(r, \psi, 2 \pi)\} d \mu(r t, \theta)+\frac{1}{t^{2}} \int_{0}^{2 \pi} \int_{0}^{\infty} \lambda(r, \psi, 2 \pi) d \mu(r t, \theta)=I_{1}+I_{2} .
$$

Now we see that

$$
I_{1}=\frac{1}{t^{2}} \int_{0}^{2 \pi} \int_{0}^{\infty}\left\{\int_{\theta}^{2 \pi} \int_{r}^{\infty} \frac{\partial^{2} \lambda}{\partial r \partial \theta} d r d \theta\right\} d \mu(r t, \theta)
$$

and, by means of the Fubini theorem or an extension of a result due to Tautz (11), we can perform a double integration by parts to obtain

$$
I_{1}=\int_{0}^{2 \pi} \int_{0}^{\infty} \frac{\mu(r t, \theta)}{(r t)^{2}} r^{2} \frac{\partial^{2} \lambda}{\partial r \partial \theta} d r d \theta
$$

where $\mu(r, \theta)$ is as defined in (2.1). The hypotheses of Theorem 3 imply that for all $r$ and $\theta$

$$
\frac{\mu(r t, \theta)}{(r t)^{2}} \rightarrow 0, \quad\left|\frac{\mu(r t, \theta)}{(r t)^{2}}\right| \leqslant B<\infty
$$

as $t \rightarrow 0+$, and we can apply the Lebesgue 'dominated convergence' theorem to (3.4) to conclude that

$$
I_{1} \rightarrow 0
$$

as $t \rightarrow 0+$. An integration by parts in $I_{2}$ yields

$$
I_{2}=-\int_{0}^{\infty} \frac{\mu(r t, 2 \pi)}{(r t)^{2}} r^{2} \frac{\partial \lambda}{\partial r} d r
$$

With (3.5) we can apply the Lebesgue theorem to (3.7) to conclude that

$$
I_{2} \rightarrow 0
$$

as $t \rightarrow 0+$. Since (3.6) and (3.8) imply (3.3) the proof is complete.

\section{Lemmas required for Theorem 4}

We list here a few results which will be used in the proof of Theorem 4. We begin with some definitions which allow us to state a form of the Wiener Tauberian theorem. (See (5) 294-9.)

Definitions. All functions are real-valued and defined over $0 \leqslant t<\infty$. We say that $g(t)$ is in $M$ if $g(t)$ is continuous and if

$$
\sum_{n=-\infty}^{\infty} \max _{e^{n} \leqslant t \leqslant e^{n+1}}|\operatorname{tg}(t)|<\infty
$$


We say that $\gamma(t)$ is in $V$ if $\gamma(t)$ has bounded variation over each finite interval and if

$$
\int_{i}^{e t} \frac{|d \gamma(r)|}{r}
$$

is bounded for $t>0$. We say that $\gamma(t)$ is in $N D$ if there exists a function $\delta(t)$ such that $\gamma(t)+\delta(t)$ is non-decreasing in $t$ and such that

$$
\frac{\delta(t)}{t} \rightarrow D
$$

as $t \rightarrow 0+$.

LEmma 2. Suppose that $g(t)$ is in $M$, that

$$
\int_{0}^{\infty} g(t) t^{i y} d t \neq 0
$$

for all real $y$, that $\gamma(t)$ is in $V$ and $N D$, and that $\gamma(0)=0$. If

$$
\frac{1}{t} \int_{0}^{\infty} g(r / t) d \gamma(r) \rightarrow C \int_{0}^{\infty} g(r) d r
$$

as $t \rightarrow 0+$, then

$$
\frac{\gamma(t)}{t} \rightarrow C
$$

as $t \rightarrow 0+$.

Undoubtedly this form of the Wiener theorem is known. However, we could find no reference and so we sketch a proof here.

Proof. After an elementary change of variable, we can conclude from a well-known theorem ((5), Theorem 234) that for each $h(t)$ in $M$

$$
\frac{1}{t} \int_{0}^{\infty} h(r / t) d \gamma(r) \rightarrow C \int_{0}^{\infty} h(r) d r
$$

as $t \rightarrow 0+$. With $h(t)=e^{-t}$ and $s=1 / t$ in (4.5) we obtain

$$
\int_{0}^{\infty} e^{-r s} d \gamma(r) \sim \frac{C}{s}
$$

as $s \rightarrow+\infty$. Since $\gamma(t)$ is in $N D$ we can find $\delta(t)$ such that $\gamma(t)+\delta(t)$ is nondecreasing and such that (4.3) holds. Now from (4.6), (4.3), and an Abelian theorem for the Laplace transform ((14) 182) we conclude that

$$
\int_{0}^{\infty} e^{-r s} d\{\gamma(r)+\delta(r)\} \sim \frac{C+D}{s}
$$


as $s \rightarrow+\infty$. The fact that $\gamma(t)+\delta(t)$ is non-decreasing, (4.7), and a wellknown Tauberian theorem ((14) 192) yield

$$
\frac{\gamma(t)+\delta(t)}{t} \rightarrow C+D
$$

as $t \rightarrow 0+$, and the desired conclusion follows from (4.8) and (4.3).

Remark. Lemma 2 also holds when $g(t)$ is real-valued and $\gamma(t)$ is complexvalued if the real and imaginary parts of $\gamma(t)$ are in $V$ and $N D$ and if $\gamma(0)=0$.

Lemma 3. Suppose that $\omega(t)$ has bounded variation and that $\omega(t)=O\left(t^{2}\right)$ for small $t$. Then

$$
\frac{\omega(t)}{t^{2}} \rightarrow L
$$

as $t \rightarrow 0+$ if and only if

$$
\frac{1}{t} \int_{0}^{t} \frac{d \omega(r)}{r} \rightarrow 2 L
$$

as $t \rightarrow 0+$.

The hypotheses imply that the improper integral in (4.9) exists in the Cauchy sense and the proof for the lemma closely follows one given by Allen and Kerr ((1) 83).

Definition. We say that a sequence of functions is pseudo equicontinuous if the limit of each convergent subsequence is continuous.

LEMma 4. Suppose that $\nu_{k}(\theta)$ is a sequence of pseudo equicontinuous functions which have uniformly bounded variation over $0 \leqslant \theta \leqslant 2 \pi$. If $\nu_{k}(0)=0$ and if for $n=0, \pm 1, \pm 2, \ldots$

as $k \rightarrow \infty$, then

$$
\int_{0}^{2 \pi} e^{i n \theta} d \nu_{k}(\theta) \rightarrow 0
$$

as $k \rightarrow \infty$ for $0 \leqslant \theta \leqslant 2 \pi$.

$$
\nu_{k}(\theta) \rightarrow 0
$$

Proof. If the lemma is false we can use the Helly theorem to find a convergent subsequence with a continuous limit $\nu(\theta) \not \equiv 0$. But (4.10) implies that

$$
\int_{0}^{2 \pi} e^{i n \theta} d \nu(\theta)=0
$$

for $n=0, \pm 1, \pm 2, \ldots$, and it follows that $\nu(\theta) \equiv 0$.

Finally, before proceeding to the proof of Theorem 4, we must evaluate the integral

$$
I(n, \psi, z)=\int_{0}^{\infty}\left\{\int_{0}^{2 \pi} \lambda(t, \psi, \theta) \cos n \theta d \theta\right\} t^{1+z} d t
$$

where $\lambda(t, \psi, \theta)$ is as defined in (3.2). 
LEMMA 5. Suppose that $0<\psi<\frac{1}{2} \pi$, that $n=1,2, \ldots$, and that $z$ is complex with $-1<\operatorname{re}(z)<1$. Then for $z \neq 0$,

$$
I(n, \psi, z)=-\frac{\pi}{\sin \pi z} P_{z}^{n+1}(0) P_{z}^{-n}(\cos \psi),
$$

where $P_{\nu}^{\mu}(\cos \theta)$ is the associated Legendre function of the first kind. (See (7) 227.) When $z=0$,

$$
I(n, \psi, 0)=\left(\tan \frac{1}{2} \psi\right)^{n} .
$$

In particular $I(n, \psi, i y) \neq 0$ for all real $y$.

Proof. Since both sides of (4.11) are analytic in $-1<\mathrm{re}(z)<1$ we can assume that $-1<\operatorname{re}(z)<0$. Next consider the iterated integral

$$
J(n, \psi, z)=\frac{1}{2 \pi} \int_{0}^{2 \pi}\left\{\int_{0}^{\infty} \frac{t^{z} d t}{\left(t^{2}-2 t \sin \psi \cos \theta+1\right)^{\frac{3}{3}}}\right\} \cos n \theta d \theta .
$$

The inner integral is a Mellin transform and can be evaluated by tables ((2) 310$)$ or by contour integration. Then appealing to the general addition theorem for Legendre functions ((7) 382) we obtain

$$
J(n, \psi, z)=-\frac{\pi}{\sin \pi z} P_{z}^{n}(0) P_{z}^{-n}(\cos \psi) .
$$

An alternative proof for (4.14) can be obtained as follows. Invert the order of integration in (4.13), expand the integrand in powers of $t$, and then evaluate the inner integral by means of the addition theorem for Legendre polynomials. Formula (4.14) can then be obtained from a formula due to Dougall ((10) 328).

Now by writing $\cos n \theta=\cos (n+1) \theta \cos \theta+\sin (n+1) \theta \sin \theta$ we can express $I(n, \psi, z)$ as the sum of two integrals and, after an integration by parts in the second integral, we obtain

The identity

$$
I(n, \psi, z)=\frac{d}{d \psi} J(n+1, \psi, z)+(n+1) \cot \psi J(n+1, \psi, z) .
$$

$$
\frac{d}{d \psi}\left\{(\sin \psi)^{n} P_{z}^{-n}(\cos \psi)\right\}=(\sin \psi)^{n} P_{z}^{-n+1}(\cos \psi)
$$

is an immediate consequence of the Mehler Dirichlet formula and (4.11) follows directly from (4.14), (4.15), and (4.16). Let $z \rightarrow 0$ in (4.11) to obtain (4.12).

Finally the conclusion that $I(n, \psi, i y) \neq 0$ for all real $y$ follows from (4.11), (4.12) and a well-known theorem due to Macdonald ((7) 403). 


\section{Proof for Theorem 4}

We can assume that $k=0$ in (1.1) and, by virtue of (1.3), that $d \mu=0$ outside of some circle $\xi^{2}+\eta^{2} \leqslant \epsilon^{2}$. The proof is in two steps. First we use an elementary argument to show that for all $\alpha<\beta$

$$
\varlimsup \frac{1}{\pi t^{2}} \int_{\alpha}^{\beta} \int_{0}^{t} d \mu(r, \theta) \leqslant(\beta-\alpha) A C
$$

as $t \rightarrow 0+$, where $C$ is an absolute constant. Then we apply the Wiener Tauberian theorem to refine (5.1) and conclude that

$$
\lim \frac{1}{\pi t^{2}} \int_{\alpha}^{\beta} \int_{0}^{t} d \mu(r, \theta)=\frac{\beta-\alpha}{2 \pi} A
$$

as $t \rightarrow 0+$ or that $D^{*} \mu(0,0)=A$.

In the proof of (5.1) we may clearly assume that $A>0$, that $\alpha=-\beta$, and that $0<\beta<1$. Since $\mu$ is non-negative (3.1) yields

$$
u(t, \psi, 0) \geqslant \frac{1}{t^{2}} \int_{-\beta}^{\beta} \int_{(1-\beta) t}^{t} \lambda(r / t, \psi, \theta) d \mu(r, \theta),
$$

where $\lambda(r, \psi, \theta)$ is as defined in (3.2). If we fix $0<\psi<\frac{1}{2} \pi$ so that

$$
1-\sin \psi=\beta^{2},
$$

it is not difficult to show that

$$
\lambda(r, \psi, \theta) \geqslant\left(\pi C \beta^{2}\right)^{-1}
$$

for $1-\beta \leqslant r \leqslant 1$ and $-\beta \leqslant \theta \leqslant \beta$. From (5.3) and (5.4) we conclude that

$$
\frac{1}{\pi} \int_{-\beta(1-\beta) t}^{\beta} \int_{-i}^{t} d \mu(r, \theta) \leqslant C \beta^{2} t^{2} u(t, \psi, 0) \leqslant 2 A C \beta^{2} t^{2}
$$

for $0<t<\delta$. Theorem 2 implies that $D \mu(0,0)=A$ and with (5.5) we obtain

$$
\begin{aligned}
\frac{1}{\pi} \int_{-\beta}^{\beta} \int_{0}^{t} d \mu(r, \theta) & =\frac{1}{\pi} \sum_{n=0}^{\infty} \int_{-\beta}^{\beta} \int_{(1-\beta)^{n+1} t}^{(1-\beta)^{n t}} d \mu(r, \theta) \\
& \leqslant 2 A C \beta^{2} t^{2} \sum_{n=0}^{\infty}(1-\beta)^{2 n}<(2 \beta) A C t^{2}
\end{aligned}
$$

for $0<t<\delta$ and this completes the proof for (5.1).

For the proof of (5.2) fix $0<\psi<\frac{1}{2} \pi$. Then by the hypotheses of Theorem 5

$$
u(t, \psi, \phi) \rightarrow A
$$

as $t \rightarrow 0+$ boundedly (Lemma 6 ) in $\phi$ and we see for $n= \pm 1, \pm 2, \ldots$ that

$$
\int_{0}^{2 \pi} u(t, \psi, \phi) e^{i n \phi} d \phi \rightarrow 0
$$


as $t \rightarrow 0+$. From (3.1) we have

where we set

$$
\int_{0}^{2 \pi} u(t, \psi, \phi) e^{i n \phi} d \phi=\frac{1}{t} \int_{0}^{\infty} g_{n}(r / t) d \gamma_{n}(r),
$$

$$
\begin{aligned}
& g_{n}(t)=t \int_{0}^{2 \pi} \lambda(t, \psi, \theta) e^{i n \theta} d \theta=t \int_{0}^{2 \pi} \lambda(t, \psi, \theta) \cos n \theta d \theta \\
& \gamma_{n}(t)=\int_{0}^{2 \pi} \int_{0}^{t} \frac{e^{i n \theta}}{r} d \mu(r, \theta) .
\end{aligned}
$$

We must now show, for each $n$, that $g_{n}(t)$ and $\gamma_{n}(t)$ satisfy the hypotheses in Lemma 2. Since $g_{n}(t)$ is continuous in $0 \leqslant t<\infty$ and $g_{n}(t)=O\left(t^{-2}\right)$ for large $t, g_{n}(t)$ satisfies (4.1) and is in $M((5) 299)$. Furthermore, by Lemma 5,

$$
\int_{0}^{\infty} g_{n}(t) t^{i y} d t=I(|n|, \psi, i y) \neq 0
$$

for all real $y$. Next consider $\gamma_{n}^{R}(t)$, the real part of $\gamma_{n}(t)$. By (5.1)

$$
\int_{i}^{e t} \frac{\left|d \gamma_{n}^{R}(r)\right|}{r} \leqslant \frac{1}{t^{2}} \int_{0}^{2 \pi} \int_{0}^{e t} d \mu(r, \theta) \leqslant B<\infty
$$

for all small $t>0$ and hence for all $t>0$. Thus $\gamma_{n}^{R}(t)$ is in $V$. Since

$$
\begin{gathered}
D \mu(0,0)=A, \\
\frac{1}{t^{2}} \int_{0}^{2 \pi} \int_{0}^{t} d \mu(r, \theta) \rightarrow \pi A
\end{gathered}
$$

as $t \rightarrow 0+$ and hence by Lemma 3

$$
\frac{\delta(t)}{t}=\frac{1}{t} \int_{0}^{2 \pi} \int_{0}^{t} \frac{d \mu(r, \theta)}{r} \rightarrow 2 \pi A
$$

as $t \rightarrow 0+$. Since $\mu$ is non-negative the sum

$$
\gamma_{n}^{R}(t)+\delta(t)=\int_{0}^{2 \pi} \int_{0}^{t} \frac{1+\cos n \theta}{r} d \mu(r, \theta)
$$

is non-decreasing in $t$ and thus $\gamma_{n}^{R}(t)$ is in $N D$. The same arguments hold for the imaginary part of $\gamma_{n}(t)$.

From Lemma 2, (5.6) and (5.7), we conclude for $n= \pm 1, \pm 2, \ldots$, that

$$
\frac{\gamma_{n}(t)}{t}=\frac{1}{t} \int_{0}^{2 \pi} \int_{0}^{t} \frac{e^{i n \theta}}{r} d \mu(r, \theta) \rightarrow 0
$$


as $t \rightarrow 0+$. By Lemma 3 we see that

$$
\frac{1}{t^{2}} \int_{0}^{2 \pi} \int_{0}^{t} e^{i n \theta} d \mu(r, \theta) \rightarrow 0
$$

as $t \rightarrow 0+$ or alternatively that

$$
\int_{0}^{2 \pi} e^{i n \theta} d \nu_{l}(\theta) \rightarrow 0
$$

as $t \rightarrow 0+$, where $\quad \nu_{t}(\theta)=\frac{1}{\pi t^{2}} \int_{0}^{\theta} \int_{0}^{t} d \mu(r, \theta)-\frac{A \theta}{2 \pi}$.

Now (5.9) holds for $n= \pm 1, \pm 2, \ldots$ by virtue of $(5.8)$; since $D \mu(0,0)=A$ it also holds for $n=0$. We see from (5.1) that the functions $\nu_{t}(\theta)$ are pseudo equicontinuous and have uniformly bounded variation in $0 \leqslant \theta \leqslant 2 \pi$ and we conclude from Lemma 4 that

$$
\nu_{l}(\theta) \rightarrow 0
$$

as $t \rightarrow 0+$ for $0 \leqslant \theta \leqslant 2 \pi$. Since (5.10) implies (5.2) the proof is complete.

\section{Extension of Theorem 4}

We can state Theorem 4 in a slightly stronger form if we make use of some results due to Tsuji, Deny, and Lelong.

Defintition. Let $R$ be a closed Jordan region on the unit hemisphere $x^{2}+y^{2}+z^{2}=1, z>0$, and let $C$ be the boundary of $R$. The set of rays

$$
\Gamma \equiv\{(t x, t y, t z) \mid(x, y, z) \in R, t>0\}
$$

will be called the cone determined by $R$, and the set of rays

$$
\Sigma \equiv\{(t x, t y, t z) \mid(x, y, z) \in C, t>0\}
$$

will be called the surface of $\Gamma$.

LEMMA 6. Suppose that $u(x, y, z)$ is in $H^{+}$and that

$$
u(x, y, z)=O(1)
$$

as $(x, y, z) \rightarrow(0,0,0)$ along some ray in $z>0$. Then for each cone $\Gamma$

$$
u(x, y, z)=O(1)
$$

uniformly as $(x, y, z) \rightarrow(0,0,0)$ in $\Gamma$.

Proof. For each $0<\psi_{0}<\frac{1}{2} \pi$ set

$$
B=\operatorname{lub} \frac{\lambda\left(t, \psi_{1}, \phi_{1}\right)}{\lambda\left(t, \psi_{2}, \phi_{2}\right)}
$$


where the upper bound is taken over all $\phi_{1}, \phi_{2}, 0 \leqslant \psi_{1}, \psi_{2} \leqslant \psi_{0}$, and $0 \leqslant t<\infty$. Obviously $1 \leqslant B<\infty$ and from (3.1) we obtain

$$
u\left(t, \psi_{1}, \phi_{1}\right) \leqslant B u\left(t, \psi_{2}, \phi_{2}\right)
$$

whenever $0 \leqslant \psi_{1}, \psi_{2} \leqslant \psi_{0}$. Lemma 6 follows directly from (6.1).

We can now prove the following extension of Theorem 4. (Cf. (9), Theorem 1.)

TheOREM 5. Suppose that $u(x, y, z)$ in $H^{+}$is given by (1.1) and that

$$
u(x, y, z) \rightarrow A
$$

as $(x, y, z) \rightarrow(0,0,0)$ along each ray in a collection of rays which is dense in the surface of some cone. Then $D^{*} \mu(0,0)=A$.

Proof. For each $0<\psi_{0}<\frac{1}{2} \pi, u(t, \psi, \phi)$ is uniformly bounded in $\phi$, $0 \leqslant \psi \leqslant \psi_{0}$, and $0<t \leqslant 1$. Let

$$
M(\psi, \phi)=\varlimsup i m u(t, \psi, \phi) \geqslant \underline{\lim } u(t, \psi, \phi)=m(\psi, \phi)
$$

as $t \rightarrow 0+$. By a theorem due to Tsuji ((12) 20), $M(\psi, \phi)$ and $m(\psi, \phi)$ are continuous, $M(\psi, \phi)$ is subharmonic, and $m(\psi, \phi)$ is superharmonic on the unit hemisphere. By hypothesis there exists a Jordan curve $C$ and a set $E$, on the unit hemisphere, such that $E$ is dense in $C$ and such that

$$
M(\psi, \phi)=A=m(\psi, \phi)
$$

for $(1, \psi, \phi) \in E$. Since $M(\psi, \phi)$ and $m(\psi, \phi)$ are continuous (6.3) holds for $(1, \psi, \phi) \in C$ and hence

$$
M(\psi, \phi) \leqslant A \leqslant m(\psi, \phi)
$$

for $(1, \psi, \phi) \in R$, the region bounded by $C$. From (6.2) and (6.4) it follows that

$$
u(x, y, z) \rightarrow A
$$

as $(x, y, z) \rightarrow(0,0,0)$ along each ray in $\Gamma$, the cone determined by $R$. Using an argument due to Tsuji (13), or alternatively a theorem due to Deny and Lelong ((3) 104), we see that (6.5) holds as $(x, y, z) \rightarrow(0,0,0)$ along each ray in $z>0$. The desired conclusion then follows from Theorem 4 .

\section{REFERENCES}

1. A. C. Allen and E. KerR, 'The converse of Fatou's theorem', J. London Math. Soc. 28 (1953) 80-89.

2. Bateman Manuscript Project, Tables of integral transforms, vol. 1 (McGraw-Hill, 1954).

3. J. Deny and P. Lelong, 'Etude des fonctions sousharmoniques dans un cylindre ou dans un cône', Bull. Soc. Math. France 75 (1947) 89-112.

4. F. W. Gehring, 'The Fatou theorem and its converse', Trans. Amer. Math. Soc. 85 (1957) 106-21.

5. G. H. Hardy, Divergent series (Oxford, 1949).

6. - and J. E. Litruewood, 'On Tauberian theorems', Proc. London Math. Soc. 30 (1930) 23-37. 
7. E. W. Hobson, Spherical and ellipsoidal harmonics (Cambridge, 1931).

8. J. Lelong-Ferrand, 'Sur le principe de Julia-Carathéodory et son extension à l'espace d̀ $p$ dimensions', Bull. Sci. Math. (2) 73 (1949) 5-16.

9. L. H. Looms, 'The converse of the Fatou theorem for positive harmonic functions', Trans. Amer. Math. Soc. 53 (1943) 239-50.

10. T. M. MAcRoBert, Spherical harmonics (Methuen, 1947).

11. G. TAUTZ, 'Eine Verallgemeinerung der partiellen Integration; uneigentliche mehrdimensionale Stieltjesintegrale', Jber. Deutsch. Math. Verein. 53 (1943) $136-46$.

12. M. TsuJI, 'On the limits of indetermination of bounded harmonic functions', Jap. J. Math. 15 (1939) 19-26.

13. ' ' On a positive harmonic function in a half-plane', J. Math. Soc. Japan 7 (1955) 76-78.

14. D. V. WIDDER, The Laplace transform (Princeton, 1946).

15. A. Zyamund, Trigonometrical series (Warsaw, 1935).

\section{University of Michigan \\ Ann Arbor, Michigan}

